\title{
Cooperative Evaporation in Ordered Arrays of Volatile Droplets
}

\author{
C. Schäfle, ${ }^{1}$ C. Bechinger, ${ }^{1}$ B. Rinn, ${ }^{1}$ C. David,${ }^{2}$ and P. Leiderer ${ }^{1}$ \\ ${ }^{1}$ Fakultät für Physik, Universität Konstanz, D-78457 Konstanz, Germany \\ ${ }^{2}$ Laboratory for Micro- and Nanotechnology, Paul Scherrer Institute, CH-5232 Villigen-PSI, Switzerland
}

(Received 23 April 1999)

\begin{abstract}
We study the evaporation behavior of regular arrays of volatile droplets on a solid substrate. We observe that under certain conditions the droplets do not evaporate independently of each other but in a cooperative manner. This results in the development of a superlattice which is explained in terms of matter exchange between adjacent droplets.
\end{abstract}

PACS numbers: 64.70.Fx, 05.70.Ln, 64.70.Rh

When bulk mixtures are rapidly quenched below their critical point (e.g., by lowering the temperature) phase separation occurs which leads to an isotropic, disordered morphology of the coexisting phases. This process is initiated by the formation of small nuclei in a supersaturated solution. When approaching local equilibrium, larger supercritical droplets grow at the expense of smaller ones thus causing the first peak in the structure factor to be shifted to smaller values. The latter is also known as Ostwald ripening or coarsening [1] and is experimentally observed in many systems like fluid mixtures, binary metal alloys [2], polymers [3], and colloidal systems [4].

Lifshitz, Slyosov, and, independently, Wagner (LSW) $[5,6]$ pointed out that for small degrees of supersaturation, when the density of nucleated droplets is small, a singledroplet picture can be used to describe the late stage growth of the condensate in an infinite system. Even at small supersaturations, however, many experiments of initially disordered droplets show that the late-stage droplet distribution is broader and more symmetric than predicted by LSW [7]. In order to understand this behavior, it has been suggested that additional interactions between the droplets have to be taken into account [7-9].

Lacasta et al. [10] investigated theoretically the kinetics and pattern formation emerging during the evaporation of periodically arranged droplets. Based on numerical solutions of the Cahn-Hilliard equation they found that initial equal-sized droplets of a volatile liquid which are arranged in a quasi-one-dimensional (1D) system with open (absorbing) boundaries do not evaporate independently of each other, but in a cooperative process by matter exchange through the gas phase. This leads to the remarkable effect that rows of droplets which are farther away from the absorbing boundary may evaporate earlier than those at the boundary's vicinity. The effect is explained in terms of a complex interplay of matter loss through the boundary and its redistribution between droplets.

Here we report the first experimental observation of such a cooperative droplet evaporation. Our results show that under appropriate geometrical constraints a periodic array of initially equal-sized droplets decays into a twodimensional (2D) regular superlattice where every second droplet disappears by evaporation. This phenomenon is also observed in 2D numerical solutions of the CahnHilliard equation, which were carried out in addition.

Surfaces with well-defined adsorption sites for droplets were obtained by microcontactprinting $(\mu \mathrm{CP})$ of alkanethiols. Since there is a vast amount of literature on $\mu \mathrm{CP}$ [11] we will refer to this technique only in brief. Stamps were fabricated by casting the elastomer poly(dimethylsiloxane) onto a silicon wafer which was topographically structured by electron-beam lithography. As substrates we used $30 \mathrm{~nm}$ gold films vacuum deposited on glass slides. We stamped for $2 \mathrm{~s}$ with a $2.5 \mathrm{mM}$ ethanolic solution of $\mathrm{HS}\left(\mathrm{CH}_{2}\right)_{11}-\mathrm{OH}$, an $\mathrm{OH}$-terminated thiol, which resulted in well-defined hydrophilic sites corresponding to the protruding parts of the stamp [12]. The formation of a thiol monolayer should be essentially completed under these conditions [13]. Afterwards the substrate was dipped into a $2.5 \mathrm{mM}$ ethanolic solution of $\mathrm{HS}\left(\mathrm{CH}_{2}\right)_{17} \mathrm{CH}_{3}$, a $\mathrm{CH}_{3}$-terminated thiol, for $\approx 3 \mathrm{~min}$ to make the remaining bare gold surface hydrophobic. The chemical contrast of the samples was controlled to be in agreement with the stamp pattern by lateral force microscopy [11]. The experiments were performed under ambient conditions (about $40 \%$ humidity) in a closed cell with diethylene glycol as test liquid because it has a relatively low vapor pressure of 0.01 mbar [14].

After diethylene glycol was quenched from supersaturated vapor onto the substrate we obtained an array of well-ordered, equal-sized volatile droplets reflecting the geometry of the underlying hydrophilic surface pattern. In order to characterize the temporal change in the adsorbate structures during the evaporation process we used an optical microscope (dark field illumination in transmission) with a CCD camera connected to a video recorder.

Figure 1(a) shows a typical section of the hexagonally shaped array of droplets with $65 \mu \mathrm{m}$ side length. The bright and dark areas correspond to the droplets and the unwetted substrate, respectively. In the following we will refer to this geometry as "honeycomb pattern." The radius of the droplets' contact line was determined to $0.75 \mu \mathrm{m}$ and the next neighbor distance to $2.5 \mu \mathrm{m}$. Outside the hexagonal region where the substrate is not patterned but 

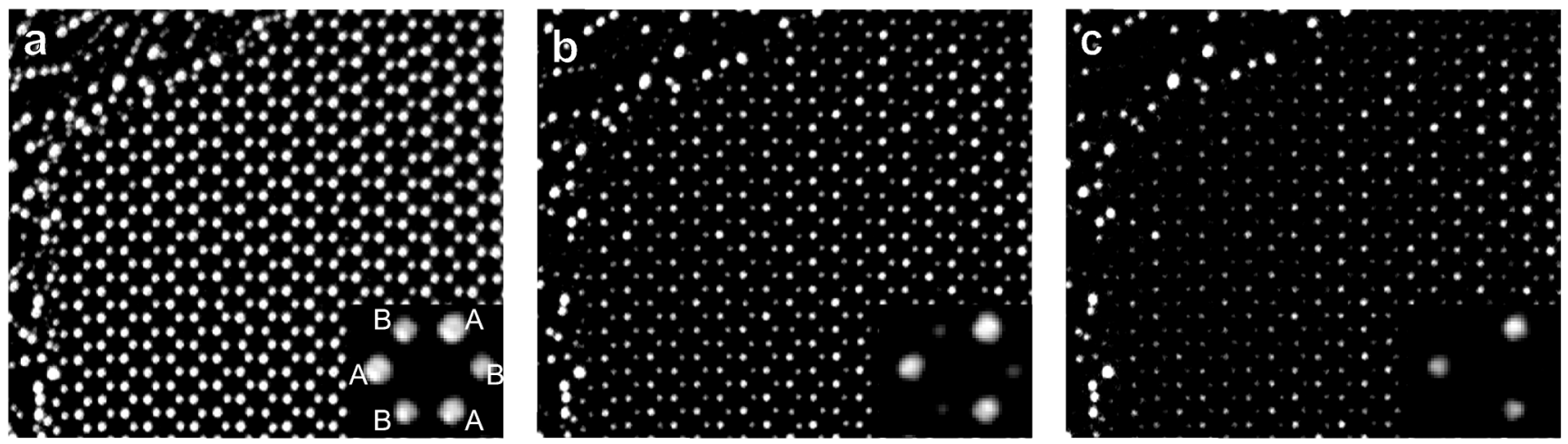

FIG. 1. Optical microscope pictures (dark field illumination) $\left(77.2 \times 66.8 \mu \mathrm{m}^{2}\right)$ of the temporal evolution of an ordered array of diethylene glycol droplets (bright) on a gold substrate (dark). Photographs were taken immediately (a), 405 s (b), and 445 s (c) after the liquid was adsorbed onto the surface. The insets correspond to a magnified area of the corresponding pictures and clearly demonstrate that droplets $A$ remain almost constant in size, whereas droplets $B$ shrink considerably as time proceeds.

homogeneously hydrophobic, the droplets are adsorbed statistically. Figure 1(b) shows the sample about $405 \mathrm{~s}$ after the diethylene glycol had been adsorbed. In contrast to Fig. 1(a) where all the droplets had identical diameters, this is no longer the case in Fig. 1(b). This becomes even more pronounced $40 \mathrm{~s}$ later in Fig. 1(c), where some of the droplets became considerably smaller while others did not change noticeably in size. A closer analysis reveals that the positions of smaller and larger droplets are not irregular, but that the initial honeycomb pattern changed into a triangular superlattice during evaporation. Obviously, the droplets within the patterned area of the sample do not evaporate independently of each other, but cooperatively as already suggested by Lacasta et al. [10]. Outside the structured region no regular pattern formation was observed [Figs. 1(a) $-1(\mathrm{c})]$.

In order to quantify the temporal development of the superlattice structure, we measured the average light intensity of the droplets at the superlattice sites $(A)$ and those at the remaining sites $(B)$ independently of each other during the evaporation process. This was done by an image processing system. The intensities $I_{A}$ and $I_{B}$ are related to the size of the droplets at the corresponding sites; therefore the ratio $I_{R}=I_{B} / I_{A}$ can be used to decide whether the droplets evaporate independently of each other or not. If the droplets evaporate in an uncorrelated manner, $I_{R}$ will remain close to 1; otherwise significant deviations are expected. A typical example of the temporal behavior of $I_{R}$ is plotted in Fig. 2. At the beginning of the experiment $(t=0) I_{R}$ is close to 1 because all the droplets have about the same size. During the first $360 \mathrm{~s}$ this value changes only slightly which indicates that the droplet size ratio remains quite stable during this interval. As can be seen, it is only in the last $90 \mathrm{~s}$ of the experiment that $I_{R}$ suddenly decreases to about 0.68 , i.e., the droplets at sites $A$ become larger compared to those at sites $B$ and the superlattice is formed. Finally all the droplets disappear by evaporation.

The formation of superlattices does not seem to be restricted to particular distances $l$ and contact radii $a$ of the droplets. It was also observed for honeycomb structures with $l=2,1.7 \mu \mathrm{m}$ and $a=0.7,0.5 \mu \mathrm{m}$, respectively. Similar results were also obtained for ethylene glycol with a roughly 10 times higher vapor pressure than that of diethylene glycol [14].

Before we turn to a theoretical model, which shows the same effect in 2D, we suggest a simple mechanism to understand the origin of the observed cooperative droplet evaporation. This model starts with two prerequisites: the long-range character of the concentration diffusional field and the vapor pressure of curved surfaces.

Droplets of volatile liquids can mutually interact by exchanging matter through the gas phase. To estimate the range over which sessile evaporating droplets can exchange matter by diffusion one has to calculate how the vapor pressure $p$, i.e., the concentration profile $c(r)$, around a sessile droplet of a radius of curvature of $R_{0}$ and contact angle $\Theta$, decays laterally. Supposing a steady state regime, $c(r)$ fulfills a Laplace equation $(\Delta c=0)$. It was already pointed out by Maxwell (see, e.g., Ref. [15]) that there is an analogy between $c(r)$ and the electrostatic potential of a spherical cap shaped electrode on a conducting

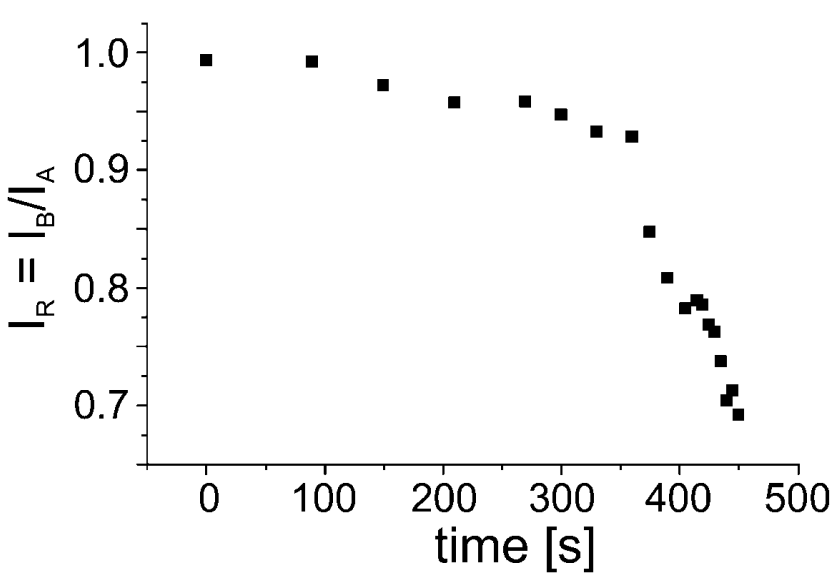

FIG. 2. Temporal behavior of $I_{R}=I_{B} / I_{A}$. The decrease of the signal after about $360 \mathrm{~s}$ corresponds to a change in the geometry of the droplet pattern from a honeycomb to a triangular lattice. The error of the data points is less than $5 \%$. 
surface. Consequently, when considering only the first term in a multipole expansion one finds $c(r)-c_{0} \propto \frac{1}{r}$, with $c_{0}$ being the background concentration.

The vapor pressure $p$ at the liquid/gas interface is well known to depend on its curvature. For a droplet of radius $R_{0}$ this is described by the Kelvin equation $\ln \frac{p\left(R_{0}\right)}{p_{\infty}}=\frac{2 \gamma V_{m}}{R_{0} R T}$, where $p_{\infty}$ is the vapor pressure above the plane surface, $\gamma$ is the liquid's surface tension, $V_{m}$ is the molar volume, $R$ is the gas constant, and $T$ is the temperature [16]. Accordingly, a large droplet in the vicinity of a smaller one will increase in size at the expense of the smaller one which eventually disappears. This scenario is very similar to the above mentioned LSW coarsening mechanism where the radius of small, initially supercritical condensate droplets can get surpassed by the critical radius as time proceeds; i.e., they become subcritical and eventually die out.

For simplicity, we will limit this qualitative explanation to a $1 \mathrm{D}$ case but the picture can be extended to 2D (as shown below). Let us assume several sessile droplets of initially equal size with an open (absorbing) boundary at the left side (dashed line) which is characterized by the saturated vapor pressure $p_{\infty}$ (Fig. 3). Immediately after the formation of droplets, there is a diffusional flux of matter as indicated by the grey arrows [Fig. 3(a)]. While the droplets far away from the boundary are in quasiequilibrium (regarding gain and loss of matter), this is not the case for the leftmost droplet. There is an effective flow of matter from this droplet across the boundary (black arrow) which leads to a reduction of the radius of droplet $B$ [Fig. 3(b)]. This, however, brings droplet $B$ out of balance with respect to the vapor pressure of $A$ [cf. Eq. (1)] and thus causes a net flow from $B$ to $A$. As a consequence, $A$ starts to grow (at the expense of $B$ ) which in turn reduces its vapor pressure and therefore leads to an effective mass flow from $B^{\prime}$ to $A^{\prime}$ [Fig. 3(c)]. This again leads to a shrinking of $B^{\prime}$ and the growth of $A^{\prime}$ and so on [Fig. 3(d)]. Thus, by exchange of matter between next neighbors, one obtains an instability (here

a)

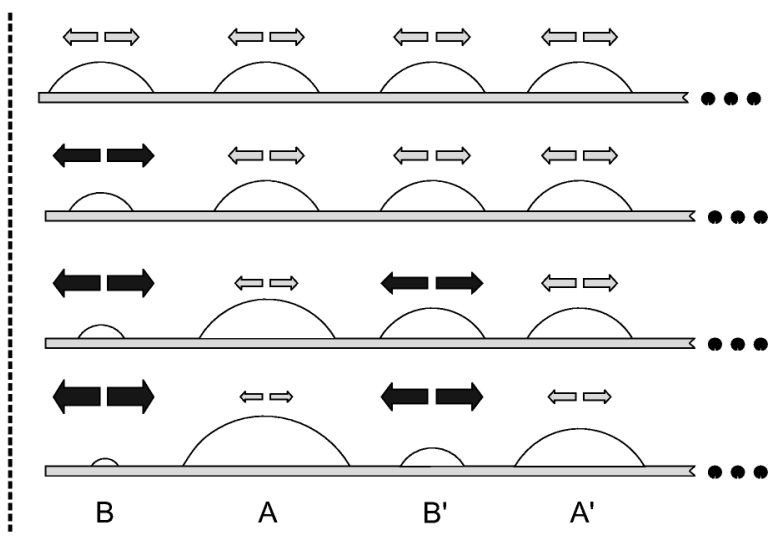

FIG. 3. Sketch of the pattern formation by cooperative evaporation of a droplet array in the vicinity of a wall. The arrows denote the net mass exchange by evaporation. induced by a boundary) which can proceed over the whole system. It can be shown by geometrical considerations that a square lattice or a honeycomb pattern (the latter used in our experiments) is commensurate with this mechanism to take place in $2 \mathrm{D}$.

In reality the situation is more complicated as there is also evaporation of matter in the vertical direction which takes place on top of the matter exchange between droplets as described above. Therefore it is only the relative size of droplets which changes upon the cooperative evaporation process while the absolute radii of both kinds of droplets decrease due to evaporative losses.

In principle the effect should also occur on other length scales, although the degree of supersaturation becomes smaller with increasing droplet size. Furthermore, at larger absolute distances one has to take into account the increased time required for lateral matter exchange which then may compete with the vertical diffusion, the latter determining the lifetime of a droplet. Therefore, and because of the reduced number of pinning centers, this makes experiments on a small length scale favorable.

So far we have assumed that a droplet always reduces its radius upon evaporation as suggested by Young's equation. The situation, however, is more complex on patterned surfaces [17]. If the volume $V$ of a droplet on a circular hydrophilic patch is so large that it covers the whole patch, its contact line is pinned to the circular boundary. Accordingly, a reduction in $V$ may lead to a larger radius of curvature and thus - in contrast to the above-would cause larger droplets to decrease at the profit of smaller ones and lead to monodisperse droplet arrays. Only when the droplets do not cover the whole hydrophilic patch, their contact angles are constant as described by Young's equation and thus cause their radii to become smaller upon evaporation which allows the above described effect to occur.

We assume that we initially prepared our system in a state where the contact lines of the droplets are fixed. The system then starts to evaporate and finally reaches a situation where all droplets have (due to the stabilization process described above) more or less the same size. During this process which may take several minutes (depending on the initial amount of adsorbed liquid) the droplets are expected to remain about equal in size (cf. Fig. 2). Only when the droplets pass a critical size they enter the regime of constant contact angle and the cooperative evaporation should occur. Accordingly the development of superlattices should take place only in the last part of the experiment where the droplets are smaller than the supporting hydrophilic patches. This scenario agrees well with the temporal behavior of $I_{R}$, which has been discussed above (cf. Fig. 2). As the superlattice formation is induced by the boundaries the underlying geometrical pattern was chosen in a way that the outermost droplets correspond to sites $B$.

It has already been pointed out by Lacasta et al. [10] that the phenomenon of cooperative droplet evaporation can 

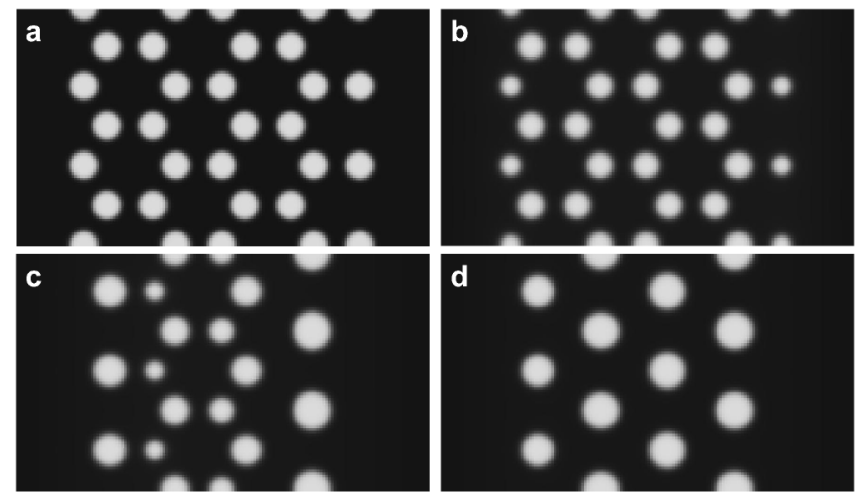

FIG. 4. Time evolution of the concentration profile as calculated by a 2D Cahn-Hilliard equation.

be qualitatively described by a concentration field $c(\boldsymbol{r}, t)$, with the liquid phase corresponding to $c=1$ and the vapor phase corresponding to $c=0$. The time development of this concentration field is then determined by the CahnHilliard equation:

$$
\frac{\partial c(\boldsymbol{r}, t)}{\partial t}=\frac{D}{2} \Delta[2 c(1-2 c)(1-c)-\Delta c],
$$

which is given here in a dimensionless form, and the diffusion parameter set to unity in the following. In the $x$ direction we use absorbing boundary conditions, which means physically that the system is in equilibrium with the saturated vapor ( $\mu$ is the chemical potential),

$$
c\left(\boldsymbol{r}_{B}, t\right)=\mu\left(\boldsymbol{r}_{B}, t\right)=0 \quad \forall t, \boldsymbol{r}_{B} \text { of the boundary. }
$$

While Lacasta et al. treated a quasi-1D system, we take into account a 2D coupling between the droplets. To do this we start from a honeycomb pattern of concentration profiles. In the $y$ direction we use periodic boundary conditions.

In order to treat Eqs. (1) and (2) numerically, we discretize the spatial degrees of freedom to second order using symmetric difference operators. The resulting system of ordinary differential equations (ODEs) is stiff due to the influence of the discrete $\Delta^{2}$ operator. To avoid the stability problems of the usual Runge-Kutta-Fehlberg methods, we utilize a BDF (backward difference formula) solver [18] to get numerical solutions of the ODE system. Because of the good stability properties and step-size control of the BDF solver we can easily handle 2D systems and continue the calculation to long times [19]. In Fig. 4 the time development of the calculated 2D concentration profile is shown. One recognizes as in the experiment the development of a triangular superlattice in an intermediate time regime. Owing to the limited size of our simulation box, further superstructures could not be observed. However, a Fourier analysis of a triangular lattice of criticaldroplets by Burghaus [20] indicates that certain rows may grow while others shrink.

Finally we want to point out that superlattice formation may not only be induced by an absorbing boundary but also by local defects, e.g., a missing droplet inside the pattern. Numerically, this was achieved by prepar- ing a closed system with periodic boundary conditions in both directions and a small, but finite density of defects. We observed that also in this case superlattice structures commensurate with the initial defects were formed. Additionally, the process observed here may also have consequences for the design of gas sensors comprised of liquid droplet arrays which are sensitive to environmental conditions, e.g., humidity [21,22].

In summary, we have investigated the kinetics and pattern formation during the evaporation process of ordered droplet arrays. We find that under appropriate conditions the droplets do not disappear independently of each other but evaporate in a cooperative process, which leads to the formation of a superlattice structure. This is to our knowledge the first direct experimental evidence for spatial correlations during Ostwald ripening in a quasi-3D-system [9]. The same effect is found in numerical solutions of a 2D Cahn-Hilliard equation. Our results confirm that the observed development of superlattice structures is quite generic and should also occur in other systems like polymer mixtures or binary metal alloys.

We gratefully acknowledge stimulating discussions with K. Zahn, P. Maaß, and R. Bubeck, and financial support from the Deutsche Forschungsgemeinschaft, SFB 513.

[1] J. A. Marqusee and John Ross, J. Chem. Phys. 79, 373 (1983).

[2] See, e.g., M. Marder, Phys. Rev. A 36, 858 (1987).

[3] Y. Oono and M. Bahiana, Phys. Rev. Lett. 61, 1109 (1988).

[4] E. K. Hobbie, Phys. Rev. Lett. 81, 3996 (1998).

[5] I. M. Lifshitz and V. V. Slyozov, J. Chem. Phys. Solids 19, 35 (1961).

[6] C. Wagner, Z. Elektrochem. 65, 581 (1961).

[7] P. W. Vorhees, J. Stat. Phys. 38, 231 (1985).

[8] M. Marder, Phys. Rev. Lett. 55, 2953 (1985); Phys. Rev. A 36, 858 (1987).

[9] O. Krichevsky and J. Stavans, Phys. Rev. Lett. 70, 1473 (1993); Phys. Rev. E 52, 1818 (1995).

[10] A. M. Lacasta, I. M. Sokolov, J. M. Sancho, and F. Sagués, Phys. Rev. E 57, 6198 (1998).

[11] A. Kumar and G. M. Whitesides, Appl. Phys. Lett. 63, 2002 (1993); A. Kumar, H. A. Biebuyck, and G. M. Whitesides, Langmuir 10, 1498 (1994).

[12] C. D. James et al., Langmuir 14, 741 (1998).

[13] E. Delamarche et al., J. Phys. Chem. B 102, 3324 (1998).

[14] D. R. Lide, Handbook of Chemistry and Physics (CRC Press LLC, Boca Raton, FL, 1998), 79th ed.

[15] R. Burghaus, Phys. Rev. E 54, 6955 (1996).

[16] J. N. Israelachvili, Intermolecular and Surface Forces (Academic Press Limited, London, 1985), 1st ed., p. 223.

[17] P. Lenz and R. Lipowsky, Phys. Rev. Lett. 80, 1920 (1998).

[18] The BDF method is an implicit, linear multistep procedure for solving stiff ODE systems.

[19] R. Kenzler et al. (to be published).

[20] R. Burghaus (to be published).

[21] A. Kumar et al., Acc. Chem. Res. 28, 219 (1995).

[22] F. Morhard et al., Proc. ECS 97-19, 1058 (1997). 